\author{
Research Article
}

Serena Dipierro, Andrea Pinamonti* and Enrico Valdinoci

\title{
Classification of stable solutions for boundary value problems with nonlinear boundary conditions on Riemannian manifolds with nonnegative Ricci curvature
}

https://doi.org/10.1515/anona-2018-0013

Received October 30, 2017; accepted January 16, 2018

\begin{abstract}
We present a geometric formula of Poincaré type, which is inspired by a classical work of Sternberg and Zumbrun, and we provide a classification result of stable solutions of linear elliptic problems with nonlinear Robin conditions on Riemannian manifolds with nonnegative Ricci curvature. The result obtained here is a refinement of a result recently established by Bandle, Mastrolia, Monticelli and Punzo.
\end{abstract}

Keywords: Riemannian manifolds, elliptic problems, Robin condition

MSC 2010: 58J05, 58J32, 53C24

\section{Introduction}

The study of partial differential equations on manifolds has a long tradition in analysis and geometry, see, e.g., $[1,26,28,32,33]$. The interest for such topic may come from different perspectives. On the one hand, at a local level, classical equations with variable coefficients can be efficiently comprised into the manifold setting, allowing more general and elegant treatments. In addition, at a global level, the geometry of the manifold can produce new interesting phenomena and interplay with the structure of the solutions, thus creating a novel scenario for the problems into consideration.

Of course, given the complexity of the topic, the different solutions of a given partial differential equation on a manifold can give rise to a rather wild "zoology" and it is important to try to group the solutions into suitable "classes" and possibly to classify all the solutions belonging to a class.

In this spirit, very natural classes of solutions in a variational setting arise from energy considerations. The simplest class in this framework is probably that of "minimal solutions", namely the class of solutions which minimize (or, more generally, local minimize) the energy functional.

On the other hand, it is often useful to look at a more general class than minimal solutions, that is, the class of solutions at which the second derivative of the energy functional is nonnegative. These solutions are

\footnotetext{
Serena Dipierro, Department of Mathematics and Statistics, University of Western Australia, 35 Stirling Highway, Crawley, WA-6009 Perth, Australia; and Dipartimento di Matematica "Federigo Enriques", Università degli studi di Milano,

Via Saldini 50, 20133 Milano, Italy, e-mail: serena.dipierro@unimi.it

*Corresponding author: Andrea Pinamonti, Dipartime nto di Matematica, Università di Trento, Via Sommarive 14, 38123 Povo, Trento, Italy, e-mail: andrea.pinamonti@gmail.com

Enrico Valdinoci, Department of Mathematics and Statistics, University of Western Australia, 35 Stirling Highway, Crawley, WA-6009 Perth, Australia; Dipartimento di Matematica "Federigo Enriques", Università degli studi di Milano, Via Saldini 50, 20133 Milano, Italy; School of Mathematics and Statistics, University of Melbourne, Grattan Street, Parkville, VIC-3010 Melbourne, Australia; and Istituto di Matematica Applicata e Tecnologie Informatiche, Via Ferrata 1, 27100 Pavia, Italy, e-mail: enrico.valdinoci@unimi.it
} 
called "stable" (see, e.g., [14]). Of course, the class of stable solutions contains that of minimal solutions, but the notion of stability is often in concrete situations more treatable than that of minimality. For instance, it is typically very difficult to establish whether or not a given solution is minimal, since one, in principle, should compare its energy with that of all the possible competitors, while a stability check could be more manageable, relying only on a single, and sometimes sufficiently explicit, second derivative bound.

The goal of this paper is to study the case of a linear elliptic equation on a domain of a Riemannian manifold with nonnegative Ricci curvature, endowed with nonlinear boundary data. We will consider stable solutions in this setting and provide sufficient conditions to ensure that they are necessarily constant.

The framework in which we work is the following. Let $M$ be a connected $m$-dimensional Riemannian manifold endowed with a smooth Riemannian metric $g=\left(g_{i j}\right)$. We denote by $\Delta$ the Laplace-Beltrami operator induced by $g$. Let $\Omega \subset M$ be a compact orientable domain and $v$ be the outer normal vector of $\partial \Omega$ lying in the tangent space $T_{p} M$ for any $p \in \partial \Omega$. We assume that $\partial \Omega$ is orientable for the outer normal to be well defined and continuous.

In this paper we study the solutions to the following boundary value problem:

$$
\begin{cases}\Delta u+f(u)=0 & \text { in } \Omega, \\ \partial_{v} u+h(u)=0 & \text { on } \partial \Omega,\end{cases}
$$

where $f, h \in C^{1}(\mathbb{R})$ and $\partial_{v} u:=g(\nabla u, v)$. Similar problems have been investigated in $[2,3,11,25]$.

As usual, we consider the volume term induced by $g$, that is, in local coordinates,

$$
d V=\sqrt{|g|} d x^{1} \wedge \cdots \wedge d x^{m},
$$

where $\left\{d x^{1}, \ldots, d x^{m}\right\}$ is the basis of 1 -forms dual to the vector basis $\left\{\partial_{1}, \ldots, \partial_{m}\right\}$, and $|g|=\operatorname{det}\left(g_{i j}\right) \geq 0$. We also denote by $d \sigma$ the volume measure on $\partial \Omega$ induced by the embedding $\partial \Omega \hookrightarrow M$.

As customary, we say that $u$ is a weak solution to (1.1) if $u \in C^{1}(\bar{\Omega})$ and

$$
\int_{\Omega}\langle\nabla u, \nabla \varphi\rangle d V+\int_{\partial \Omega} h(u) \varphi d \sigma=\int_{\Omega} f(u) \varphi d V \quad \text { for any } \varphi \in C^{1}(\Omega) .
$$

Moreover, we say that a weak solution $u$ is stable if

$$
\int_{\Omega}|\nabla \varphi|^{2} d V+\int_{\partial \Omega} h^{\prime}(u) \varphi^{2} d \sigma-\int_{\Omega} f^{\prime}(u) \varphi^{2} d V \geq 0 \quad \text { for any } \varphi \in C^{1}(\Omega) .
$$

In order to state our result, we recall below some classical notions in Riemannian geometry. Given a vector field $X$, we set

$$
|X|=\sqrt{\langle X, X\rangle} .
$$

Also (see, for instance, [26, Definition 3.3.5]), it is customary to define the Hessian of a smooth function $\phi$ as the symmetric 2-tensor given in a local patch by

$$
\left(H_{\phi}\right)_{i j}=\partial_{i j}^{2} \phi-\Gamma_{i j}^{k} \partial_{k} \phi,
$$

where $\Gamma_{i j}^{k}$ are the Christoffel symbols, namely,

$$
\Gamma_{i j}^{k}=\frac{1}{2} g^{h k}\left(\partial_{i} g_{h j}+\partial_{j} g_{i h}-\partial_{h} g_{i j}\right) .
$$

Given a tensor $A$, we define its norm by $|A|=\sqrt{A A^{*}}$, where $A^{*}$ is the adjoint.

The above quantities are related to the Ricci tensor Ric via the Bochner-Weitzenböck formula (see, for instance, [4] and references therein):

$$
\frac{1}{2} \Delta|\nabla \phi|^{2}=\left|H_{\phi}\right|^{2}+\langle\nabla \Delta \phi, \nabla \phi\rangle+\operatorname{Ric}(\nabla \phi, \nabla \phi) .
$$

Finally, we let II and $H$ denote the second fundamental tensor and the mean curvature of the embed$\operatorname{ding} \partial \Omega \hookrightarrow \Omega$ in the direction of the outward unit normal vector field $v$, respectively. 
We are now in position to state our main result.

Theorem 1.1. Let $u \in C^{3}(\bar{\Omega})$ be a stable solution to (1.1). Assume that the Ricci curvature is nonnegative in $\Omega$, and that

(C1) for any $p \in \partial \Omega$, the quadratic form $\mathbb{I}-h^{\prime}(u) \tilde{g}$ on the tangent space $T_{p}(\partial \Omega)$ is nonpositive definite.

If

$$
\int_{\partial \Omega}\left(h(u) f(u)+(m-1)(h(u))^{2} H+h^{\prime}(u)(h(u))^{2}\right) d \sigma \leq 0,
$$

then $u$ is constant in $\Omega$.

Remark 1.2. Theorem 1.1 has been proved in [2, Theorem 4.5] in the particular case where $h(t):=\alpha t$ for some $\alpha \in \mathbb{R}$. We point out that with this particular choice of $h$, Theorem 1.1 here weakens the assumptions on the sign of $\alpha$ of [2, Theorem 4.5].

The proof of Theorem 1.1 is based on a geometric Poincaré-type inequality, which we state in this setting as follows.

Theorem 1.3. Let $u$ be a stable weak solution to (1.1). Then

$$
\int_{\Omega}\left(\operatorname{Ric}(\nabla u, \nabla u)+\left|H_{u}\right|^{2}-\left.|\nabla| \nabla u\right|^{2}\right) \varphi^{2} d V-\int_{\partial \Omega}\left(\frac{1}{2}\left\langle\nabla|\nabla u|^{2}, v\right\rangle+h^{\prime}(u)|\nabla u|^{2}\right) \varphi^{2} d \sigma \leq \int_{\Omega}|\nabla u|^{2}|\nabla \varphi|^{2} d V
$$

for any $\varphi \in C^{\infty}(\Omega)$.

We notice that formula (1.5) relates the stability condition of the solution with the principal curvatures and the tangential gradient of the corresponding level set. Since this formula bounds a weighted $L^{2}$-norm of any $\varphi \in C^{1}(\Omega)$ plus a boundary term by a weighted $L^{2}$-norm of its gradient, we may consider this formula as a weighted Poincaré type inequality.

The idea of using weighted Poincaré inequalities to deduce quantitative and qualitative information on the solutions of a partial differential equation has been originally introduced by Sternberg and Zumbrun in $[30,31]$ in the context of the Allen-Cahn equation, and it has been extensively exploited to prove symmetry and rigidity results, see, e.g., $[15,18,19]$. See also [16, 20, 21, 23, 24, 29] for applications to Riemannian and sub-Riemannian manifolds, [7] for problems involving the Ornstein-Uhlenbeck operator, [6, 17] for semilinear equations with unbounded drift and [8-10, 22] for systems of equations.

Recently, in $[11,13]$, the cases of Neumann conditions for boundary reaction-diffusion equations and of Robin conditions for linear and quasilinear equations have been studied, using a Poincaré inequality that involves also suitable boundary terms.

We point out that Theorem 1.1 comprises the classical case of the Laplacian in the Euclidean space with homogeneous Neumann data, which was studied in the celebrated papers [5, 27]. In this spirit, our Theorem 1.1 can be seen as a nonlinear version of the results of $[5,27]$ on Riemannian manifolds (and, with respect to [5, 27], we perform a technically different proof, based on Theorem 1.3).

For related results in the framework of Markov Triples, see [12]. The next two sections are devoted to the proofs of Theorems 1.3 and 1.1, respectively.

\section{Proof of Theorem 1.3}

Applying (1.2) with $\varphi$ replaced by $\left|\nabla_{g} u\right| \varphi$, we get

$$
\begin{aligned}
\int_{\Omega} f^{\prime}(u)|\nabla u|^{2} \phi^{2} d V & \leq \int_{\Omega}\left(\left.|\nabla| \nabla u\right|^{2} \phi^{2}+|\nabla u|^{2}|\nabla \phi|^{2}+2 \phi|\nabla u|\langle\nabla \phi, \nabla|\nabla u|\rangle\right) d V+\int_{\partial \Omega} h^{\prime}(u)|\nabla u|^{2} \varphi^{2} d \sigma \\
& =\int_{\Omega}\left(\left.|\nabla| \nabla u\right|^{2} \phi^{2}+|\nabla u|^{2}|\nabla \phi|^{2}+\frac{1}{2}\left\langle\nabla \phi^{2}, \nabla|\nabla u|^{2}\right\rangle\right) d V+\int_{\partial \Omega} h^{\prime}(u)|\nabla u|^{2} \varphi^{2} d \sigma .
\end{aligned}
$$


Therefore, integrating by parts, the third term in the last line, we get

$$
\begin{aligned}
& \int_{\Omega} f^{\prime}(u)|\nabla u|^{2} \phi^{2} d V \leq \int_{\Omega}\left(\left.|\nabla| \nabla u\right|^{2} \phi^{2}+|\nabla u|^{2}|\nabla \phi|^{2}-\frac{1}{2} \phi^{2} \Delta|\nabla u|^{2}\right) d V \\
&+\frac{1}{2} \int_{\partial \Omega} \varphi^{2}\left\langle\nabla|\nabla u|^{2}, v\right\rangle d \sigma+\int_{\partial \Omega} h^{\prime}(u)|\nabla u|^{2} \varphi^{2} d \sigma .
\end{aligned}
$$

Hence, recalling (1.3),

$$
\begin{gathered}
\int_{\Omega} f^{\prime}(u)|\nabla u|^{2} \phi^{2} d V \leq \int_{\Omega}\left[\left.|\nabla| \nabla u\right|^{2} \phi^{2}+|\nabla u|^{2}|\nabla \phi|^{2}-\left(\left|H_{u}\right|^{2}+\langle\nabla \Delta u, \nabla u\rangle+\operatorname{Ric}(\nabla u, \nabla u)\right) \varphi^{2}\right] d V \\
+\frac{1}{2} \int_{\partial \Omega} \varphi^{2}\left\langle\nabla|\nabla u|^{2}, v\right\rangle d \sigma+\int_{\partial \Omega} h^{\prime}(u)|\nabla u|^{2} \varphi^{2} d \sigma .
\end{gathered}
$$

Now, by differentiating the equation in (1.1), we see that

$$
-\nabla \Delta u=f^{\prime}(u) \nabla u .
$$

Plugging this information into (2.1), we conclude that

$$
\begin{gathered}
0 \leq \int_{\Omega}\left[\left.|\nabla| \nabla u\right|^{2} \phi^{2}+|\nabla u|^{2}|\nabla \phi|^{2}-\left(\left|H_{u}\right|^{2}+\operatorname{Ric}(\nabla u, \nabla u)\right) \phi^{2}\right] d V \\
+\frac{1}{2} \int_{\partial \Omega} \varphi^{2}\left\langle\nabla|\nabla u|^{2}, v\right\rangle d \sigma+\int_{\partial \Omega} h^{\prime}(u)|\nabla u|^{2} \varphi^{2} d \sigma,
\end{gathered}
$$

which completes the proof of Theorem 1.3.

\section{Proof of Theorem 1.1}

In this section we provide the proof of Theorem 1.1. We first state the following result that proves [2, Theorem 3.4] in the more general case in which $h$ is any $C^{1}$ function.

Theorem 3.1. Let $w \in C^{3}(\bar{\Omega})$ satisfy

$$
\partial_{\nu} w+h(w)=0 \quad \text { on } \partial \Omega \text {, }
$$

for some $h \in C^{1}(\mathbb{R})$. Then

$$
\frac{1}{2} \frac{\partial}{\partial v}|\nabla w|^{2}=\mathbb{I}(\tilde{\nabla} w, \tilde{\nabla} w)-h^{\prime}(w)|\tilde{\nabla} w|^{2}-h(w) H_{w}(v, v) \quad \text { on } \partial \Omega,
$$

where $\tilde{\nabla} w:=\nabla w-g(\nabla w, v) v$ is the tangential gradient with respect to $\partial \Omega$, and $H_{w}$ is the Hessian matrix of the function $w$.

Proof. We let $\left\{e_{i}\right\}$, with $i \in\{1, \ldots, m\}$, be a Darboux frame along $\partial \Omega$, that is, such that $e_{m}:=v$. In this setting, conditions (3.1) reads

$$
w_{m}=-h(w) \text { on } \partial \Omega .
$$

Also, for any $i, j \in\{1, \ldots, m-1\}$, we define

$$
H_{i j}:=g\left(\mathbb{I}\left(e_{i}, e_{j}\right), v\right) .
$$

Then, reasoning as in the proof of $[2$, formula (3.32)], we obtain that, for any $j \in\{1, \ldots, m-1\}$,

$$
w_{j m}=\sum_{i=1}^{m-1} H_{i j} w_{i}-h^{\prime}(w) w_{j} \quad \text { on } \partial \Omega .
$$


Therefore, multiplying both terms by $w_{j}$, we get

$$
w_{j m} w_{j}=\sum_{i=1}^{m-1} H_{i j} w_{i} w_{j}-h^{\prime}(w) w_{j}^{2} \quad \text { on } \partial \Omega .
$$

On the other hand, for any $i \in\{1, \ldots, m\}$,

$$
\frac{1}{2}\left(|\nabla w|^{2}\right)_{i}=\sum_{j=1}^{m} w_{j} w_{j i}=\sum_{i=1}^{m-1} w_{j} w_{j i}+w_{m} w_{m i}=\sum_{i=1}^{m-1} w_{j} w_{j i}-h(w) w_{m i},
$$

where we used (3.2) in the last passage.

As a consequence,

$$
\frac{1}{2} \frac{\partial}{\partial v}|\nabla w|^{2}=\sum_{j=1}^{m-1} w_{j} w_{j m}-h(w) w_{m m} \quad \text { on } \partial \Omega .
$$

From this and (3.3), we thus obtain

$$
\frac{1}{2} \frac{\partial}{\partial v}|\nabla w|^{2}=\sum_{i, j=1}^{m-1} H_{i j} w_{i} w_{j}-h^{\prime}(w) \sum_{j=1}^{m-1} w_{j}^{2}-h(w) w_{m m} \quad \text { on } \partial \Omega,
$$

which implies the desired result.

Now we recall that $\Delta$ is the Laplace-Beltrami operator of the manifold $(M, g)$, and we let $\tilde{\Delta}$ be the LaplaceBeltrami operator of the manifold $\partial \Omega$ endowed with the induced metric by the embedding $\partial \Omega \hookrightarrow M$. We have that

$$
\Delta w=\tilde{\Delta} w-(m-1) H \frac{\partial w}{\partial v}+H_{w}(v, v) .
$$

With this, we can prove the following result.

Lemma 3.2. Let $u \in C^{3}(\bar{\Omega})$ be a stable solution of (1.1). Then

$$
\begin{aligned}
& \int_{\Omega}\left(\operatorname{Ric}(\nabla u, \nabla u)+\left|H_{u}\right|^{2}-|\nabla| \nabla u||^{2}\right) \varphi^{2} d V \\
& \quad-\int_{\partial \Omega}\left(\mathbb{I}(\tilde{\nabla} u, \tilde{\nabla} u)-h^{\prime}(u)|\tilde{\nabla} u|^{2}+h(u) f(u)+(m-1)(h(u))^{2} H+h^{\prime}(u)(h(u))^{2}\right) \varphi^{2} d \sigma \\
& \leq \int_{\Omega}|\nabla u|^{2}|\nabla \varphi|^{2} d V-\int_{\partial \Omega} h(u)\left\langle\tilde{\nabla} u, \tilde{\nabla} \varphi^{2}\right\rangle d \sigma
\end{aligned}
$$

for any $\varphi \in C^{\infty}(\Omega)$.

Proof. From Theorems 1.3 and 3.1, for every stable weak solution $u$ to (1.1) and for any $\varphi \in C^{\infty}(\Omega)$, we have that

$$
\begin{aligned}
& \int_{\Omega}\left(\operatorname{Ric}(\nabla u, \nabla u)+\left|H_{u}\right|^{2}-\left.|\nabla| \nabla u\right|^{2}\right) \varphi^{2} d V \\
& \quad-\int_{\partial \Omega}\left(\mathbb{I}(\tilde{\nabla} u, \tilde{\nabla} u)-h^{\prime}(u)|\tilde{\nabla} u|^{2}-h(u) H_{u}(v, v)+h^{\prime}(u)|\nabla u|^{2}\right) \varphi^{2} d \sigma \\
& \leq \int_{\Omega}|\nabla u|^{2}|\nabla \varphi|^{2} d V .
\end{aligned}
$$

Now we use (3.4) to manipulate the integral on the boundary of $\Omega$. In this way, we obtain from (3.6) that

$$
\begin{aligned}
& \int_{\Omega}\left(\operatorname{Ric}(\nabla u, \nabla u)+\left|H_{u}\right|^{2}-|\nabla| \nabla u||^{2}\right) \varphi^{2} d V \\
& \quad-\int_{\partial \Omega}\left[\mathbb{I}(\tilde{\nabla} u, \tilde{\nabla} u)-h^{\prime}(u)|\tilde{\nabla} u|^{2}-h(u)\left(\Delta u-\tilde{\Delta} u+(m-1) H \frac{\partial u}{\partial v}\right)+h^{\prime}(u)|\nabla u|^{2}\right] \varphi^{2} d \sigma \\
& \leq \int_{\Omega}|\nabla u|^{2}|\nabla \varphi|^{2} d V .
\end{aligned}
$$


Thus, recalling ${ }^{1}(1.1)$, we conclude that

$$
\begin{aligned}
& \int_{\Omega}\left(\operatorname{Ric}(\nabla u, \nabla u)+\left|H_{u}\right|^{2}-|\nabla| \nabla u||^{2}\right) \varphi^{2} d V \\
& \quad-\int_{\partial \Omega}\left(\mathbb{I}(\tilde{\nabla} u, \tilde{\nabla} u)-h^{\prime}(u)|\tilde{\nabla} u|^{2}+h(u) f(u)+h(u) \tilde{\Delta} u+(m-1)(h(u))^{2} H+h^{\prime}(u)|\nabla u|^{2}\right) \varphi^{2} d \sigma \\
& \leq \int_{\Omega}|\nabla u|^{2}|\nabla \varphi|^{2} d V .
\end{aligned}
$$

Now we observe that

$$
|\nabla u|^{2}=|\tilde{\nabla} u|^{2}+\left|\frac{\partial u}{\partial v}\right|^{2}=|\tilde{\nabla} u|^{2}+(h(u))^{2} \quad \text { on } \partial \Omega .
$$

Plugging this information into (3.7), we obtain that

$$
\begin{aligned}
& \int_{\Omega}\left(\operatorname{Ric}(\nabla u, \nabla u)+\left|H_{u}\right|^{2}-|\nabla| \nabla u||^{2}\right) \varphi^{2} d V \\
& \quad-\int_{\partial \Omega}\left(\mathbb{I}(\tilde{\nabla} u, \tilde{\nabla} u)+h(u) f(u)+h(u) \tilde{\Delta} u+(m-1)(h(u))^{2} H+h^{\prime}(u)(h(u))^{2}\right) \varphi^{2} d \sigma \\
& \leq \int_{\Omega}|\nabla u|^{2}|\nabla \varphi|^{2} d V .
\end{aligned}
$$

Now we notice that

$$
\int_{\partial \Omega} h(u) \varphi^{2} \tilde{\Delta} u d \sigma=-\int_{\partial \Omega} h^{\prime}(u)|\tilde{\nabla} u|^{2} \varphi^{2} d \sigma-\int_{\partial \Omega} h(u)\left\langle\tilde{\nabla} u, \tilde{\nabla} \varphi^{2}\right\rangle d \sigma,
$$

and therefore

$$
\begin{aligned}
& \int_{\Omega}\left(\operatorname{Ric}(\nabla u, \nabla u)+\left|H_{u}\right|^{2}-|\nabla| \nabla u||^{2}\right) \varphi^{2} d V \\
& \quad-\int_{\partial \Omega}\left(\mathbb{I}(\tilde{\nabla} u, \tilde{\nabla} u)-h^{\prime}(u)|\tilde{\nabla} u|^{2}+h(u) f(u)+(m-1)(h(u))^{2} H+h^{\prime}(u)(h(u))^{2}\right) \varphi^{2} d \sigma \\
& \leq \int_{\Omega}|\nabla u|^{2}|\nabla \varphi|^{2} d V-\int_{\partial \Omega} h(u)\left\langle\tilde{\nabla} u, \tilde{\nabla} \varphi^{2}\right\rangle d \sigma,
\end{aligned}
$$

which proves the desired inequality.

Before completing the proof of Theorem 1.1 we recall the following lemmata proved in [21, Lemma 5] and [21, Lemma 9], respectively.

Lemma 3.3. For any smooth function $\phi: M \rightarrow \mathbb{R}$, we have that

$$
\left|H_{\phi}\right|^{2} \geq|\nabla| \nabla \phi||^{2} \quad \text { almost everywhere. }
$$

Lemma 3.4. Suppose that the Ricci curvature of $M$ is nonnegative and that Ric does not vanish identically. Let $u$ be a solution of (1.1), with

$$
\operatorname{Ric}(\nabla u, \nabla u)(p)=0 \quad \text { for any } p \in M
$$

Then $u$ is constant.

With this, we are able to finish the proof of Theorem 1.1.

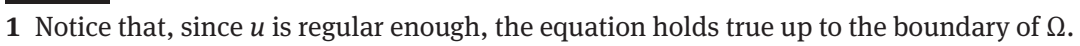


Proof of Theorem 1.1. Taking $\varphi \equiv 1$ in (3.5), we see that

$$
\begin{aligned}
& \int_{\Omega}\left(\operatorname{Ric}(\nabla u, \nabla u)+\left|H_{u}\right|^{2}-|\nabla| \nabla u||^{2}\right) d V \\
& \quad \leq \int_{\partial \Omega}\left(\mathbb{I}(\tilde{\nabla} u, \tilde{\nabla} u)-h^{\prime}(u)|\tilde{\nabla} u|^{2}+h(u) f(u)+(m-1)(h(u))^{2} H+h^{\prime}(u)(h(u))^{2}\right) d \sigma .
\end{aligned}
$$

Hence, using (C1) and (1.4), we obtain that

$$
\int_{\Omega}\left(\operatorname{Ric}(\nabla u, \nabla u)+\left|H_{u}\right|^{2}-|\nabla| \nabla u||^{2}\right) d V \leq 0 .
$$

On the other hand, by Lemma 3.3,

$$
\left|H_{u}\right|^{2}-\left.|\nabla| \nabla u\right|^{2} \geq 0 \quad \text { on } \Omega,
$$

and so (3.8) gives

$$
\int_{\Omega} \operatorname{Ric}(\nabla u, \nabla u) d V \leq 0
$$

which implies that

$$
\operatorname{Ric}(\nabla u, \nabla u)=0 \quad \text { in } \Omega .
$$

Thus, the conclusion follows from Lemma 3.4.

Funding: Part of this work was done while Andrea Pinamonti was visiting the Dipartimento di Matematica "Federigo Enriques" of the University of Milan. The authors are members of Gruppo Nazionale per l'Analisi Matematica, la Probabilità e le loro Applicazioni (GNAMPA) of the Istituto Nazionale di Alta Matematica (INdAM). Supported by the Australian Research Council Discovery Project Grant “N.E.W. Nonlocal Equations at Work”.

\section{References}

[1] T. Aubin, Some Nonlinear Problems in Riemannian Geometry, Springer Monogr. Math., Springer, Berlin, 1998.

[2] C. Bandle, P. Mastrolia, D. D. Monticelli and F. Punzo, On the stability of solutions of semilinear elliptic equations with Robin boundary conditions on Riemannian manifolds, SIAM J. Math. Anal. 48 (2016), no. 1, 122-151.

[3] C. Bandle, F. Punzo and A. Tesei, Existence and nonexistence of patterns on Riemannian manifolds, J. Math. Anal. Appl. 387 (2012), no. 1, 33-47.

[4] M. Berger, P. Gauduchon and E. Mazet, Le spectre d'une variété riemannienne, Lecture Notes in Math. 194, Springer, Berlin, 1971.

[5] R. G. Casten and C. J. Holland, Instability results for reaction diffusion equations with Neumann boundary conditions, J. Differential Equations 27 (1978), no. 2, 266-273.

[6] A. Cesaroni, M. Novaga and A. Pinamonti, One-dimensional symmetry for semilinear equations with unbounded drift, Commun. Pure Appl. Anal. 12 (2013), no. 5, 2203-2211.

[7] A. Cesaroni, M. Novaga and E. Valdinoci, A symmetry result for the Ornstein-Uhlenbeck operator, Discrete Contin. Dyn. Syst. 34 (2014), no. 6, 2451-2467.

[8] S. Dipierro, Geometric inequalities and symmetry results for elliptic systems, Discrete Contin. Dyn. Syst. 33 (2013), no. 8, 3473-3496.

[9] S. Dipierro and A. Pinamonti, A geometric inequality and a symmetry result for elliptic systems involving the fractional Laplacian, J. Differential Equations 255 (2013), no. 1, 85-119.

[10] S. Dipierro and A. Pinamonti, Symmetry results for stable and monotone solutions to fibered systems of PDEs, Commun. Contemp. Math. 17 (2015), no. 4, Article ID 1450035.

[11] S. Dipierro, A. Pinamonti and E. Valdinoci, Rigidity results for elliptic boundary value problems: Stable solutions for quasilinear equations with Neumann or Robin boundary conditions, Int. Math. Res. Not. IMRN 2018 (2018), DOI https://doi.org/10.1093/imrn/rny055.

[12] S. Dipierro, A. Pinamonti and E. Valdinoci, Rigidity results in Diffusion Markov Triples, preprint (2018), https://arxiv.org/abs/1801.00941.

[13] S. Dipierro, N. Soave and E. Valdinoci, On stable solutions of boundary reaction-diffusion equations and applications to nonlocal problems with Neumann data, Indiana Univ. Math. J. 67 (2018), no. 1, 429-469. 
[14] L. Dupaigne, Stable Solutions of Elliptic Partial Differential Equations, Chapman \& Hall/CRC Monogr. Surv. Pure Appl. Math. 143, Chapman \& Hall/CRC, Boca Raton, FL, 2011.

[15] A. Farina, Propriétés qualitatives de solutions d'équations et systèmes d'équations non-linéaires, Habilitation à diriger des recherches, Paris VI, 2002.

[16] A. Farina, L. Mari and E. Valdinoci, Splitting theorems, symmetry results and overdetermined problems for Riemannian manifolds, Comm. Partial Differential Equations 38 (2013), no. 10, 1818-1862.

[17] A. Farina, M. Novaga and A. Pinamonti, Symmetry results for nonlinear elliptic operators with unbounded drift, NoDEA Nonlinear Differential Equations Appl. 21 (2014), no. 6, 869-883.

[18] A. Farina, B. Sciunzi and E. Valdinoci, Bernstein and De Giorgi type problems: New results via a geometric approach, Ann. Sc. Norm. Super. Pisa Cl. Sci. (5) 7 (2008), no. 4, 741-791.

[19] A. Farina, B. Sciunzi and E. Valdinoci, On a Poincaré type formula for solutions of singular and degenerate elliptic equations, Manuscripta Math. 132 (2010), no. 3-4, 335-342.

[20] A. Farina, Y. Sire and E. Valdinoci, Stable solutions of elliptic equations on Riemannian manifolds with Euclidean coverings, Proc. Amer. Math. Soc. 140 (2012), no. 3, 927-930.

[21] A. Farina, Y. Sire and E. Valdinoci, Stable solutions of elliptic equations on Riemannian manifolds, J. Geom. Anal. 23 (2013), no. 3, 1158-1172.

[22] M. Fazly and N. Ghoussoub, De Giorgi type results for elliptic systems, Calc. Var. Partial Differential Equations 47 (2013), no. 3-4, 809-823.

[23] F. Ferrari and A. Pinamonti, Nonexistence results for semilinear equations in Carnot groups, Anal. Geom. Metr. Spaces 1 (2013), 130-146.

[24] F. Ferrari and E. Valdinoci, A geometric inequality in the Heisenberg group and its applications to stable solutions of semilinear problems, Math. Ann. 343 (2009), no. 2, 351-370.

[25] S. Jimbo, On a semilinear diffusion equation on a Riemannian manifold and its stable equilibrium solutions, Proc. Japan Acad. Ser. A Math. Sci. 60 (1984), no. 10, 349-352.

[26] J. Jost, Riemannian Geometry and Geometric Analysis, Universitext, Springer, Berlin, 1995.

[27] H. Matano, Asymptotic behavior and stability of solutions of semilinear diffusion equations, Publ. Res. Inst. Math. Sci. 15 (1979), no. 2, 401-454.

[28] C. B. Morrey, Jr., Multiple Integrals in the Calculus of Variations, Grundlehren Math. Wiss. 130, Springer, New York, 1966.

[29] A. Pinamonti and E. Valdinoci, A geometric inequality for stable solutions of semilinear elliptic problems in the Engel group, Ann. Acad. Sci. Fenn. Math. 37 (2012), no. 2, 357-373.

[30] P. Sternberg and K. Zumbrun, A Poincaré inequality with applications to volume-constrained area-minimizing surfaces, J. Reine Angew. Math. 503 (1998), 63-85.

[31] P. Sternberg and K. Zumbrun, Connectivity of phase boundaries in strictly convex domains, Arch. Ration. Mech. Anal. 141 (1998), no. 4, 375-400.

[32] F. W. Warner, Foundations of Differentiable Manifolds and Lie Groups, Scott Foresman, Glenview, 1971.

[33] R. O. Wells, Jr., Differential Analysis on Complex Manifolds, Prentice-Hall, Englewood, 1973. 